\title{
Análise de falhas de implantes cirúrgicos no Brasil: a necessidade de uma regulamentação adequada
}

\author{
Retrieval and failure analysis of surgical implants \\ in Brazil: the need for proper regulation
}

Cesar R. de Farias Azevedo 1

Eduardo Hippert Jr. 2

\footnotetext{
1 Laboratório de Metalografia e Análise de Falhas, Instituto de Pesquisas Tecnológicas do Estado de São Paulo. Rua Professor Almeida Prado 532, São Paulo, SP 05508-901, Brasil. crfaze@ipt.br

2 Laboratório de Mecânica da Fratura e Integridade Estrutural, Instituto de Pesquisas Tecnológicas do Estado de São Paulo. Rua Professor Almeida Prado 532, São Paulo, SP 05508-901, Brasil. hippert@ipt.br,
}

\begin{abstract}
This paper summarizes several cases of metallurgical failure analysis of surgical implants conducted at the Laboratory of Failure Analysis, Instituto de Pesquisas Tecnologicas (IPT), in Brazil. Failures with two stainless steel femoral compression plates, one stainless steel femoral nail plate, one Ti-6Al-4V alloy maxillary reconstruction plate, and five Nitinol wires were investigated. The results showed that the implants were not in accordance with ISO standards and presented evidence of corrosion-assisted fracture. Furthermore, some of the implants presented manufacturing/processing defects which also contributed to their premature failure. Implantation of materials that are not biocompatible may cause several types of adverse effects in the human body and lead to premature implant failure. A review of prevailing health legislation is needed in Brazil, along with the adoption of regulatory mechanisms to assure the quality of surgical implants on the market, providing for compulsory procedures in the reporting and investigation of surgical implants which have failed in service.
\end{abstract}

Key words Protheses and Implants; Prosthesis Failure; Legislation

Resumo Este artigo apresenta alguns casos de análise metalúrgica de falhas de implantes cirúrgicos metálicos utilizados no Brasil. Investigaram-se as causas das falhas de duas placas de compressão de aço inoxidável, uma placa-lâmina de aço inoxidável, uma placa de reconstrução de maxilar de liga de Ti com 6\% de alumínio e 4\% de vanádio (Ti-6Al-4V) e cinco arames de $\mathrm{Ni}$ tinol (liga níquel-titânio). Adicionalmente, investigou-se a conformidade destes materiais às especificações técnicas da norma ABNT (Associação Brasileira de Normas Técnica). A investigação revelou que todos os implantes analisados não estavam de acordo com os requisitos mínimos da ABNTIISO, e que as fraturas prematuras ocorreram por mecanismos assistidos por corrosão e/ou pela presença de defeitos (de fabricação, montagem ou de manuseio). Dados de literatura indicam que implantes de materiais não biocompatíveis podem causar diversos tipos de reações adversas no corpo humano, além de promover a falha prematura do componente e causar danos para o paciente e prejuízos para o investimento público. Não há no Brasil legislação sanitária que tornem compulsórios os procedimentos de notificação e de investigação dos casos de falhas de implantes cirúrgicos.

Palavras-chave Próteses e Implantes; Falha de Prótese; Legislação 


\section{Introdução}

Dados históricos mundiais revelam que entre 1940 e 1975, aproximadamente cem milhões de implantes metálicos foram colocados em seres humanos, e estima-se que com os avanços tecnológicos e maior longevidade da população mundial esses números sejam proporcionalmente maiores (Smith \& Black, 1976). Contudo, a ocorrência de falhas de implantes cirúrgicos durante o uso não é rara, e torna-se cada vez mais necessário que os usuários do sistema de saúde, os hospitais e os profissionais da área se acautelem em relação às complicações que a falha de um implante cirúrgico pode causar (Ferraz, 1997). As falhas podem ser desencadeadas por vários fatores (Tabela 1), como na seleção do material, erros de projeto, na produção e na colocação do implante; falhas de reparação do osso; ou ainda pela combinação desses fatores (Williams, 1976).

Nos Estados Unidos, a atual legislação sobre falhas de implantes é bem rigorosa, e em casos de litígio, os fabricantes são considerados responsáveis legais por seus produtos, mesmo quando eventuais falhas ou erros de fabricação não são efetivamente provados (RANDScience and Technology Policy Institute, 2000). De fato, um número crescente de ações legais, envolvendo enormes quantias, têm sido instauradas naquele país como conseqüência de implantes cirúrgicos que falharam em serviço. A disputa legal entre as partes envolvidas (paciente, médico, hospital e fabricante) é muitas vezes complexa, principalmente devido à falta de uma investigação minuciosa sobre a retirada do implante (procedimentos e ensaios de conformidade) e de documentação sobre a relação médico-paciente (Lemon, 1977).

Durante a reoperação para troca do componente defeituoso, podem ser obtidas informações fundamentais para a identificação das causas da falha do implante, desde que alguns procedimentos investigativos sejam seguidos. Nesse sentido, existe nos países desenvolvidos uma legislação sanitária adequada, mecanismos reguladores e normas técnicas específicas, como por exemplo da ASTM (American Society for Testing and Materials) e da ISO (International Organization for Standartization), para guiar os procedimentos de retirada, notificação e análise dos implantes que falharam. Estudos realizados na década de 60, nos Estados Unidos, apontavam um índice de falha em implantes metálicos de aproximadamente 5\% (Martz, 1967, apud Dumblenton \& Miller, 1975), e outra estimativa americana da década de 70 , apontava um índice inferior a $1 \%$ para os im- plantes mais comuns (Black, 1976). Dados atuais apontam, por exemplo, índice de falha em implantes de quadril em torno de $0,01 \%$. Nesse caso, um aumento do índice de falhas para $0,03 \%$ motivou o recall das peças pelas agências reguladoras dos Estados Unidos e Europa (FDA, 2001; MDA, 2001).

As normas internacionais da ASTM (F56187 - ASTM, 1987) e da ISO (12891-1 - ISO, 1999; 12891-2 - ISO, 2000a; 12891-3, ISO: 2000b; 12891-4 - ISO, 2000c), recomendam que, durante a reoperação, a equipe médica deva fotografar o local antes e depois da retirada do implante; obter amostras para exames microbiológicos e histológicos; retirar cuidadosamente o implante, preservando as superfícies danificadas e de fratura; identificar todos os componentes e promover a esterilização das peças. Recomenda-se ainda, que esses componentes sejam enviados para os ensaios de conformidade do material, análise dimensional e exame fractográfico da superfície de fratura. Emite-se, então, um laudo multidisciplinar (baseado no histórico clínico, e nos resultados dos ensaios clínicos e metalúrgicos) explicando as causas da falha prematura do implante para a respectiva agência nacional de controle sanitário (por exemplo, Food and Drug Administration - FDA, Department of Health and Human Services, nos Estados Unidos, e a Medical Devices Agency MDA - do Reino Unido) (FDA, 1996; MDA, 1998). A partir da análise minuciosa dessa documentação, a agência reguladora toma as medidas cabíveis, que vão desde simples notificações, passando pela proibição de comercialização e aplicação de multas pesadas, até a promoção de inovações tecnológicas. O Instituto Nacional de Saúde dos Estados Unidos (National Institute of Health), por exemplo, apóia atualmente, um programa de melhoria do desempenho de implantes médicos, pela criação de um banco de dados alimentado pela análise sistemática de implantes recuperados (post-mortem e em caso de falhas) (NIH, 2000).

Os implantes metálicos trabalham sob a ação de complexos carregamentos mecânicos em meio salino, que exige do material ótima resistência à corrosão. Os mecanismos de desgaste e corrosão podem promover a liberação de partículas ou de íons metálicos no corpo humano, podendo causar, além da falha prematura do implante, reações adversas aos seres humanos. Por exemplo, cromo e níquel são agentes cancerígenos no ambiente industrial e estudos realizados por pesquisadores da Universidade de Cambridge comprovaram que alguns íons metálicos solúveis (Co, Cr, Ni e Mo) são citotóxicos e produzem tumores malígnos 


\begin{tabular}{|c|c|}
\hline Etapa & Causas das falhas \\
\hline Projeto do implante & $\begin{array}{l}\text { Seção transversal insuficiente, esforços cíclicos associados à presença de regiões } \\
\text { de alta concentração de tensões (entalhes, marcações em relevo, etc.) }\end{array}$ \\
\hline Fabricação do implante & Presença de defeitos no material e no implante; falta de precisão dimensional. \\
\hline Seleção do material & $\begin{array}{l}\text { Material incompatível; propriedades físicas, magnéticas e mecânicas incompatíveis; } \\
\text { sensibilidade do paciente }\end{array}$ \\
\hline Procedimento cirúrgico & $\begin{array}{l}\text { Introdução de riscos, entalhes e deformação excessiva; danos pela utilização } \\
\text { de ferramentas impróprias; procedimento de inserção incorreto; escolha } \\
\text { de implante inadequado; uso de materiais dissimilares }\end{array}$ \\
\hline Reparação óssea & $\begin{array}{l}\text { Reparação lenta, que pode causar sobrecarga no material; afrouxamento devido } \\
\text { à reabsorção óssea }\end{array}$ \\
\hline Uso impróprio & $\begin{array}{l}\text { Acidentes com o paciente ou falta de informação sobre as restrições impostas } \\
\text { com o uso do implante. }\end{array}$ \\
\hline
\end{tabular}

em cobaias. Estudos têm mostrado ainda que pacientes com implantes totais em juntas, apresentam maior susceptibilidade ao câncer quando comparados com uma população genérica (Dora et al., 1998). Daí a importância da seleção adequada dos materiais metálicos biocompatíveis previstos em normas.

De fato, o pH dos líquidos corpóreos (meio salino) é levemente básico e gira em torno de 7,2 a 7,4, sendo que, em regiões machucadas e hematomas, o $\mathrm{pH}$ pode se tornar ácido (atingir valores próximos a 5,0 e 4,0) e, nos casos de infeções, o pH do meio pode tornar-se alcalino. A

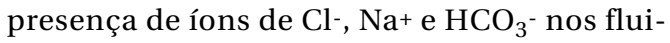
dos (celulares e sangüíneos) em contato com o implante metálico pode promover a atuação de mecanismos de corrosão do metal (Pohler, 1986).

Do ponto de vista mecânico, por exemplo, um implante de articulação de cabeça de fêmur (implante permanente) suporta cargas aplicadas que variam de três a quatro vezes o peso do paciente, e a freqüência de ciclos de esforçorepouso no implante pode chegar a três milhões ao longo de um ano (Dumblenton \& Miller, 1975). Nesses casos, a resistência à fadiga do material e as características do projeto, produção e montagem dos implantes são muito importantes. A presença de defeitos superficiais favorece a nucleação prematura de trincas de fadiga e de pontos de concentração de tensões. O crescimento das trincas é, via de regra, acelerado pelo mecanismo de corrosão, levando à falha prematura do componente por um mecanismo do tipo misto. O desgaste é a remoção mecânica de material pelo movimento relativo entre as superfícies de contato, e pode afetar o desempenho de próteses totais de articulação ou de conjuntos placas-parafusos (Dumblenton \& Miller, 1975; Pohler, 1986). Em 1995, a Academia Americana de Cirurgiões Ortopédicos afirmou que o desgaste é um fator relevante que afeta a qualidade da fixação e a durabilidade de implantes, e que os problemas advindos do desgaste podem ser responsáveis por cerca de $9 \%$ de todas as reoperações realizadas nos Estados Unidos a cada ano (Campbell et al., 1999).

As falhas de implantes temporários são mais freqüentes em tratamento de fratura do que em cirurgia corretiva, que são geralmente realizadas em condições mais controladas. De fato, as dificuldades de estabilização biomecânica do conjunto osso-implante aumentam à medida que as condições da cirurgia também vão se tornando mais complexas. Assim, a estabilização do conjunto é mais favorável em cirurgias de substituições de articulações, e vai sucessivamente tornando-se mais complicada em casos de cirurgia ortopédica corretiva, tratamentos de fratura e cirurgia de tumores (Dumblenton \& Miller, 1975; Pohler, 1986). Nos casos de fraturas com presença de intensa fragmentação, a fixação interna é mais susceptível a carregamentos complexos, o que pode causar concentração de esforços mecânicos em pontos específicos e favorecer a nucleação de trincas por fadiga ou por corrosão-fadiga. Fa- 
lhas de reparação do osso freqüentemente promovem a nucleação de trincas por fadiga no implante junto à zona de fratura. A realização de exames patológicos do osso na região da fratura durante a reoperação pode constatar a ocorrência de falhas de reparação no osso (Dumblenton \& Miller, 1975; Pohler, 1986).

Alguns casos mais complexos de falha de implantes podem ser resolvidos por uma análise biomecânica. Por exemplo, um pino intramedular colocado no fêmur direito de um rapaz de 16 anos, sofreu deformação plástica (flexão) enquanto o jovem vestia suas calças em pé (Piotrowski, 1976). O estudo metalúrgico mostrou que o material do pino estava de acordo com as especificações técnicas de composição química e de propriedades mecânicas, não tendo sido encontrados defeitos que pudessem ser associados à falha. Contudo, uma análise biomecânica deste movimento apontou que uma carga compressiva de $547 \mathrm{~N}$ e um momento fletor de 92,1 N.m teriam sido transmitidos ao longo da seção transversal da coxa. A carga compressiva é tolerável e é essencial para a reparação do osso. A carga de flexão, entretanto, está bem acima do limite de $23 \mathrm{~N}$.m calculado em projeto para o pino. Concluiu-se que a deformação plástica do pino foi conseqüência de um intenso carregamento causado pela própria ação do paciente, e esta falha não seria evitada pelo emprego de um material diferente ou de um projeto otimizado. Nesse mesmo estudo, perguntou-se a cinco médicos se, em um caso de pino intramedular em fratura de fêmur, seria permitido ao paciente vestir suas próprias calças em pé. Apenas um dos médicos considerou que essa ação do paciente não seria apropriada.

No Brasil, ainda não há uma legislação específica que regulamente a comercialização de implantes cirúrgicos, nem um procedimento para a notificação e a investigação dos casos de falha. Dessa maneira, não existem dados estatísticos que descrevam aspectos técnicos e econômicos destas falhas (gastos diretos e indiretos com reoperações, principais causas de falhas, etc.). Estudos do Instituto Nacional de Tecnologia (INT) do Rio de Janeiro (Cavalcanti \& Coelho, 1997; Cavalcanti et al., 1995), mencionam que o mau desempenho dos implantes metálicos temporários de aço inoxidável colocados no país, era decorrente da má qualidade do material utilizado. Isso elevaria o risco de corrosão em placas, pinos e parafusos, e provocaria reações inflamatórias e novas fraturas, causando prejuízos para os pacientes e para o investimento público.

\section{Procedimento experimental e resultados}

Foram analisados casos de falha de implantes metálicos encaminhados ao Instituto de Pesquisas Tecnológicas (IPT) por pacientes, médicos, fabricantes e/ou hospitais. Os componentes são listados a seguir: duas placas de compressão para fêmur fabricadas em aço inoxidável; uma placa-lâmina (nail-plate) para fêmur em aço inoxidável; um conjunto placa bucomaxilo-facial com parafusos, ambos em liga de Ti-6Al-4V, para reconstrução mandibular e cinco arames em Nitinol (liga de níquel e titânio). O trabalho experimental envolveu a inspeção visual das amostras, a caracterização macro e microestrutural do material (usando estereoscópio Karl-Zeiss Jena e microscópio Neophot), a investigação fractográfica (usando microscópio eletrônico de varredura JEOL 5200 equipado com microssonda EDS, Energy Dispersive Spectrometer) da superfície de fratura e áreas adjacentes dos componentes fraturados, e a análise da composição química de cada material (espectrômetro de fluorescência por raios$\mathrm{X}$ da Shimadzu, espectrômetro de emissão óptica por plasma da Thermo Jarrel Ash, analisador por combustão direta da LECO, espectrofotômetro de absorção atômica da Shimadzu e determinador de gases por fusão em gás inerte da LECO).

As duas placas de compressão investigadas apresentaram fratura ao longo de um dos furos (Figura 1). As superfícies chanfradas dos furos da placa próximos à fratura apresentaram características de corrosão em frestas associada à corrosão por atrito, indicando a presença de movimento relativo entre a placa e a cabeça do parafuso. O exame da superfície da cabeça de um dos parafusos apresentou as mesmas características, marcas de corrosão e desgaste nas superfícies de contato (Figura 2). A superfície final de fratura de ambas as placas de compressão apresentaram estrias de fadiga associadas com a presença de trincamento secundário (Figura 3). Os resultados indicaram que em ambos os casos a falha ocorreu por um mecanismo de corrosão-fadiga, iniciado nos pontos de atrito entre a placa e o parafuso, e propagou-se sob a ação de baixas tensões nominais em esforço de flexão unidirecional. Nas duas placas e no parafuso de fixação analisados a composição química dos materiais (Tabela 2) não atendia aos requisitos mínimos da norma NBR-ISO: 5832-1 (ABNT, 1999), indicando uma possível redução na resistência à corrosão do material. Esse tipo de falha envolvendo o movimento relativo e instabilidade mecâ- 
Exemplo da fratura encontrada na placa de compressão para fêmur fabricada em aço inoxidável (a); presença de desgaste e marcas de corrosão na superfície do buraco da placa (b).

Figura 1

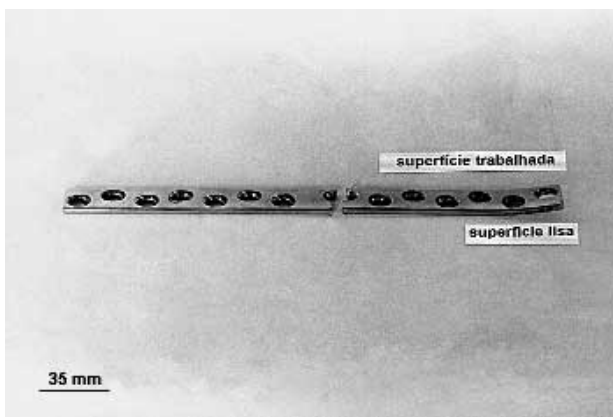

Figura $1 b$

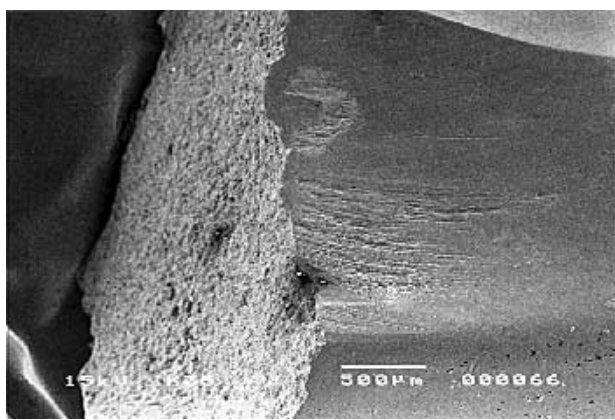

nica do conjunto osso-implante pode ser catalisada por falhas de reparação no osso, daí a importância da amostragem de material biológico durante a retirada do implante.

A fratura da placa-lâmina (com ângulo de 130) ocorreu na região da lâmina da placa que apresentava intensa redução de espessura. A lâmina apresentava uma seção transversal em $\mathrm{T}$, onde foi possível observar a presença de defeitos de manufatura, como cantos vivos, pequenas trincas e marcas de usinagem. A composição química do material (Tabela 2) não atendia aos requisitos mínimos exigidos pela norma NBR-ISO: 5832-1 (ABNT, 1999). A análise da superfície de fratura revelou duas áreas opostas com presença de estrias de fadiga, além da presença de uma pequena área central de fratura dúctil. Os resultados indicaram que
Figura 2

Cabeça do parafuso da placa de compressão mostrando (a) desgaste e (b) marcas de corrosão na superfície de contato.

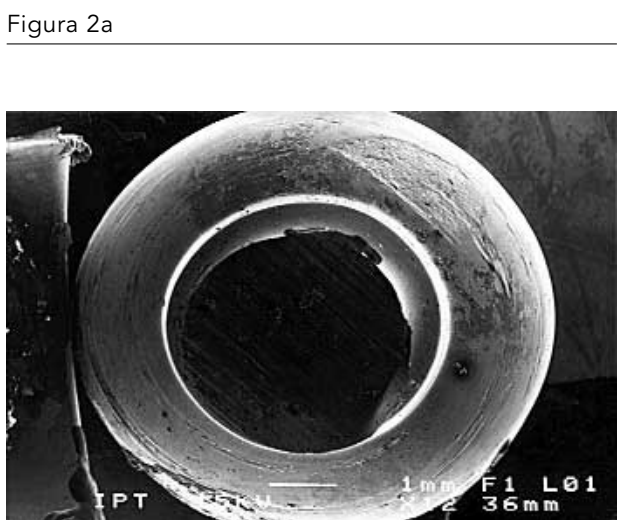

Figura $2 b$

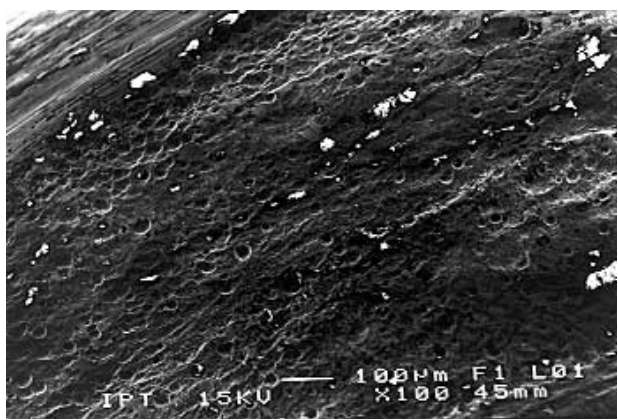

a fratura ocorreu por fadiga sob a ação de baixas tensões nominais em esforço de flexão reversa (Figura 4). A literatura aponta que falhas por fadiga em placa-pino (nail-plate) ou placalâmina (blade-plate) são invariavelmente atribuídas à instabilidade mecânica da redução da fratura, podendo decorrer de erros na instalação (Mears, 1979).

A placa buco-maxilo-facial para reconstrução mandibular fraturou ao longo de uma de suas reentrâncias, que atuam como concentradores de tensão (Figura 5a). Verificou-se que a composição química da placa (inicialmente declarada como sendo liga Ti-6Al-4V) não estava de acordo com as exigências mínimas da norma NBR-ISO: 5832-3 (ABNT, 1997b) para esta liga (Tabela 3). Entretanto, placas usadas em cirurgias buco-maxilo-faciais geralmente 
Exemplo de estrias de fadiga associadas com trincamento secundário na superfície de fratura da placa de compressão.

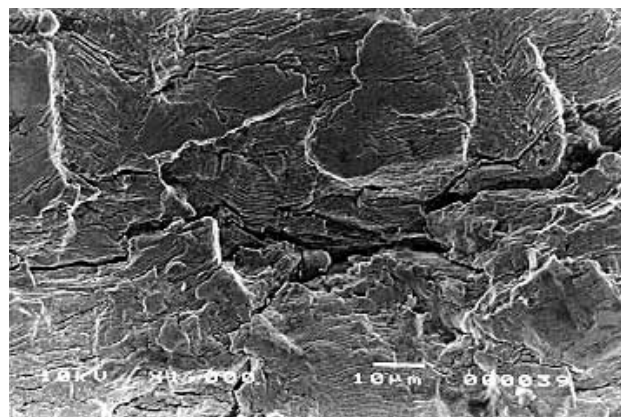

são fabricadas em ligas de titânio comercialmente puro (CP Ti), e de fato a composição química e o tamanho de grão metalúrgico da placa analisada estão de acordo com os requisitos da norma NBR-ISSO: 5832-2 (ABNT, 1997a), grau 1 (CP Ti) (Torgensen \& Gjerdet, 1995). A composição química dos parafusos se adequou, via de regra, às especificações para a liga Ti-6Al-4V. Entretanto, a montagem do conjunto placa-parafuso utilizando materiais dissimilares é contrária à boa prática, podendo favorecer a corrosão galvânica e a ocorrência de fragilização por hidrogênio na placa de CP-Ti (ISO/TR 14283 - ISO, 1995). O exame microestrutural indicou a presença de intensa precipitação de plaquetas de hidreto de titânio (de forma intra e intergranular) em região adjacente à fratura (Figura 5b), embora o teor de hidrogênio estivesse bem abaixo do valor máximo exigido pela norma ISO/TR: 14283 (ISO, 1995). A presença de hidretos é prejudicial para as propriedades mecânicas dessas ligas de Titânio (Nishigaki et al., 1980). O exame fractográfico revelou ainda a ocorrência de clivagem transgranular associada a trincamento secundário, característica típica de falha por sobrecarga em materiais frágeis (Figura $5 \mathrm{c}$ ).

As superfícies de fratura dos arames de Nitinol indicaram a ocorrência de fratura dúctil associada à grande proporção de áreas corroídas, causando a falha dos arames por sobrecarga (Figura 6). O mecanismo de corrosão foi identificado como ataque seletivo ao níquel, e foi associado à intensa presença de defeitos superficiais de fabricação nos arames, o que dificulta a formação de camada protetora de óxido (repassivação) (Schwaninger et al., 1982). Adicionalmente, a composição química dos arames de Nitinol não atende aos requisitos defi-
Figura 4

Falha de uma placa-pino para fêmur fabricada em aço inoxidável (a); presença de defeitos de fabricação, como cantos vivos e marcas de usinagem (vide seta C) (b); detalhe da superfície de fratura apresentando estrias de fadiga (c).

Figura $4 a$

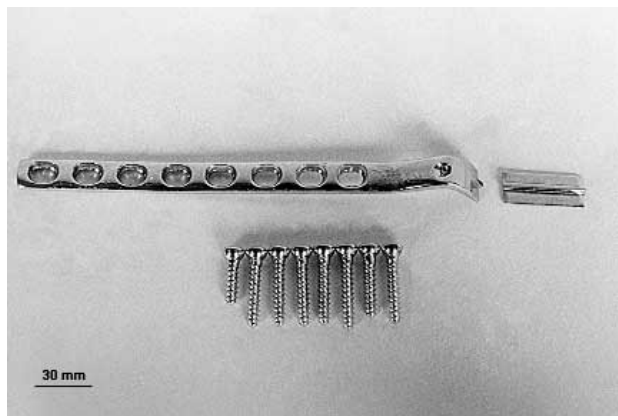

Figura $4 b$

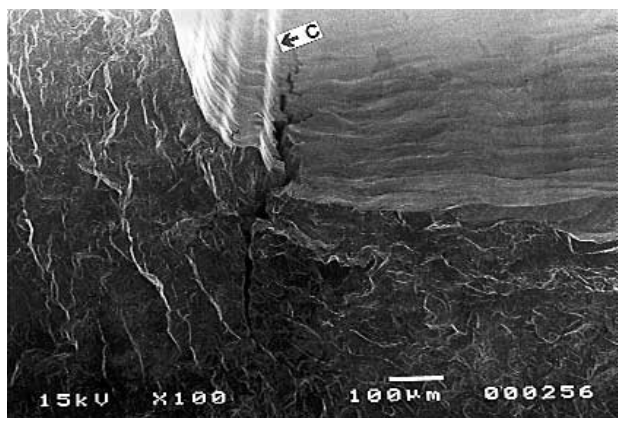

Figura $4 c$

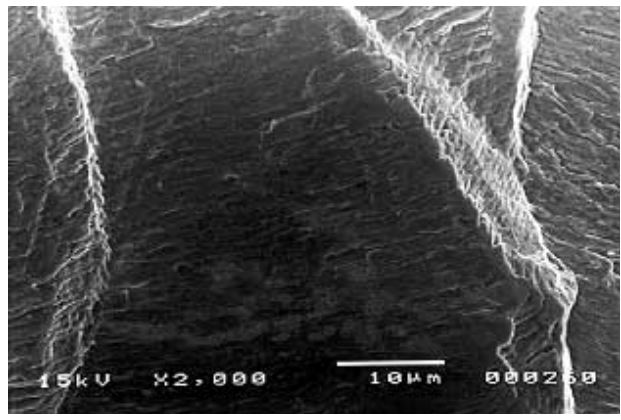


Composição química (\%) de implantes de aço inoxidável.

\begin{tabular}{|c|c|c|c|c|c|c|c|c|c|}
\hline Implante & C & $M n$ & Si & $\mathrm{Cr}$ & $\mathrm{Ni}$ & $\mathrm{S}$ & $\mathbf{P}$ & Mo & $\begin{array}{c}3,3 \% \mathrm{Mo} \\
+\mathrm{Cr}\end{array}$ \\
\hline Placa compressão 1 & 0,010 & 1,69 & 0,37 & 16,8 & 12,7 & 0,003 & 0,020 & 2,5 & 25,1 \\
\hline Placa compressão 2 & 0,010 & 1,90 & 0,56 & 17,8 & 13,9 & 0,004 & 0,020 & 2,1 & 24,7 \\
\hline Parafuso placa 2 & 0,010 & 1,80 & 0,58 & 17,2 & 11,9 & 0,004 & 0,030 & 2,1 & 23,5 \\
\hline Placa-lâmina & 0,03 & 1,89 & 0,38 & 17,5 & 13,3 & $<0,002$ & 0,02 & 2,11 & 24,5 \\
\hline ISO 5832-1 tipo D & $\begin{array}{c}0,03 \\
\text { máximo }\end{array}$ & $\begin{array}{c}2,0 \\
\text { máximo }\end{array}$ & $\begin{array}{c}1,0 \\
\text { máximo }\end{array}$ & $17,0-19,0$ & $13,0-15,0$ & $\begin{array}{r}0,010 \\
\text { máximo }\end{array}$ & $\begin{array}{r}0,025 \\
\text { máximo }\end{array}$ & $2,25-3,5$ & $\begin{array}{c}26,0 \\
\text { mínimo }\end{array}$ \\
\hline ISO 5832-1 tipo E & $\begin{array}{c}0,03 \\
\text { máximo }\end{array}$ & $\begin{array}{c}2,0 \\
\text { máximo }\end{array}$ & $\begin{array}{c}1,0 \\
\text { máximo }\end{array}$ & $17,0-19,0$ & $14,0-16,0$ & $\begin{array}{r}0,010 \\
\text { máximo }\end{array}$ & $\begin{array}{r}0,025 \\
\text { máximo }\end{array}$ & $2,25-4,2$ & $\begin{array}{c}26,0 \\
\text { mínimo }\end{array}$ \\
\hline
\end{tabular}

Tabela 3

Composição química (\%) de implantes e parafusos de ligas de Titânio e arames Nitinol.

\begin{tabular}{|c|c|c|c|c|c|c|c|c|}
\hline Dispositivo & $\mathrm{Al}$ & V & 0 & $\mathbf{N}$ & $\mathrm{C}$ & $\mathrm{H}$ & $\mathrm{Ni}$ & $\mathrm{Ti}$ \\
\hline Mini-placa Titânio & - & - & 0,09 & 0,006 & $<0,1$ & 0,003 & - & balanço \\
\hline Parafusos (média) & $4,6+1,8$ & $4,5+0,4$ & ND & ND & ND & ND & ND & balanço \\
\hline Arames de Nitinol & - & - & 0,04 & - & 0,05 & - & 55,4 & balanço \\
\hline NBR-ISO 5832-2 & - & - & $\begin{array}{c}0,45 \\
\text { máximo }\end{array}$ & $\begin{array}{c}\text { 0,05 } \\
\text { máximo }\end{array}$ & $\begin{array}{c}0,1 \\
\text { máximo }\end{array}$ & $\begin{array}{l}0,0125 \\
\text { máximo }\end{array}$ & - & balanço \\
\hline NBR-ISO 5832-3 & $5,5-6,75$ & $3,5-4,5$ & $\begin{array}{c}0,2 \\
\text { máximo }\end{array}$ & $\begin{array}{c}\text { 0,05 } \\
\text { máximo }\end{array}$ & $\begin{array}{c}0,08 \\
\text { máximo }\end{array}$ & $\begin{array}{c}0,015 \\
\text { máximo }\end{array}$ & - & balanço \\
\hline ASTM F2063-00 & - & - & $\begin{array}{c}0,05 \\
\text { máximo }\end{array}$ & - & $\begin{array}{c}0,02 \\
\text { máximo }\end{array}$ & - & $54,5-57,0$ & balanço \\
\hline
\end{tabular}

ND = não determinado

nidos pela norma ASTM: F2063-00 (ASTM, 2000) (Tabela 3). O Nitinol é um material desenvolvido na década de 60 , que apresenta propriedades interessantes como superplasticidade e biocompatibilidade, tendo várias aplicações na bioengenharia como material para ferramentas de válvulas cardíacas, arames guias para cateterismo, instrumentos de cirurgia pouco invasoras, implantes de quadril, grampos ósseos e placas de crânio (Castleman et. al., 1976; Duerig et al., 1999).

\section{Discussão}

Os maiores mercados para produtos médicohospitalares são os Estados Unidos, com aproximadamente US\$62 bilhões, seguidos da Comunidade Européia, US $\$ 40$ bilhões, do Japão, US $\$ 20$ bilhões, e países emergentes, US $\$ 15,6$ bilhões. No Brasil, este setor foi responsável no ano 2000 pela geração de 37.500 posições de trabalho e um faturamento de aproximadamente US\$3 bilhões, ocupando a sétima posição no ranking mundial, segundo dados do International Trade Data System (ITDS) - U. S. Government (ABIMO, 2001; ITDS, 2001).

Este estudo apresentou a análise de dez componentes que falharam em serviço, encaminhados ao IPT para a investigação da causa metalúrgica dessas falhas. Observou-se que nenhum dos materiais investigados obedecia aos requisitos mínimos definidos pelas normas da Associação Brasileira de Normas Técnicas (ABNT) ou ASTM (no caso dos arames de Nitinol). Além disso, dois dos implantes cirúrgicos e todos os arames ortodônticos apresentaram defeitos de fabricação (defeitos superficiais, marcas de usinagem, cantos vivos) ou de projeto (mudança brusca de seção). Deve-se res- 
Figura 5

Falha de uma placa oral maxilo-facial para reconstrução mandibular (a); detalhe da microestrutura mostrando a possível presença de hidretos de Ti precipitados ao longo dos contornos de grão e no interior dos grãos (500x) (b); detalhes da superfície de fratura revelando modo de fratura por clivagem transgranular associado a trincamento secundário (500x) (c).

Figura $5 a$

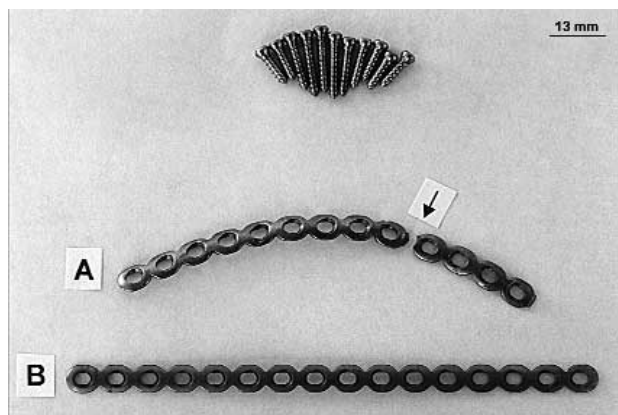

Figura $5 b$

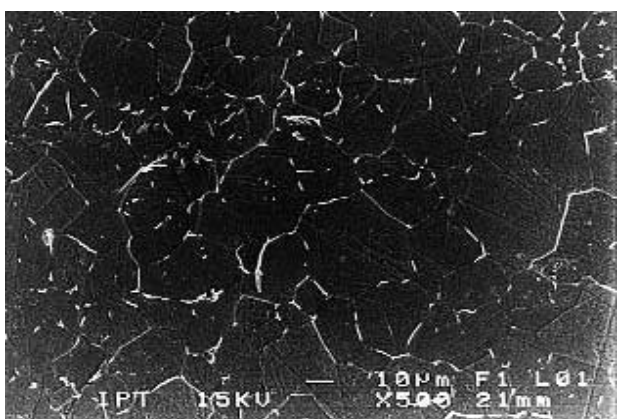

Figura $5 c$

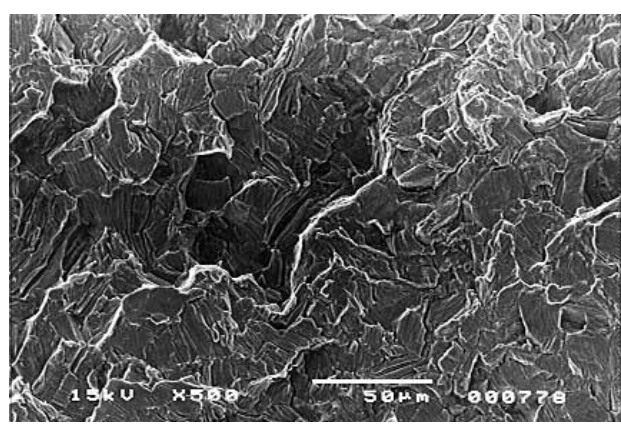

Figura 6

Arames de Nitinol (a); Visão geral da superfície de fratura mostrando defeitos superficiais e corrosão massiva (b); Detalhe da superfície de fratura mostrando fratura dúctil (c); detalhe de microestrutura acicular e evolução de trincamento lateral (150x) (d).

Figura 6a

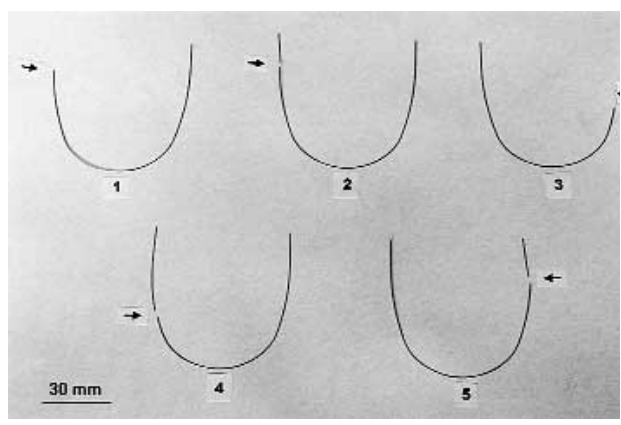

Figuras $6 b$ e $6 c$

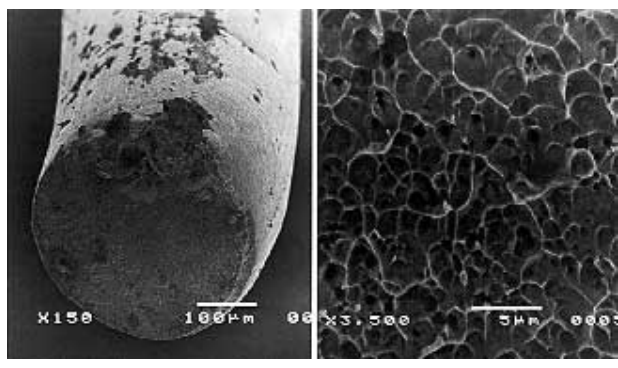

Figura 6d

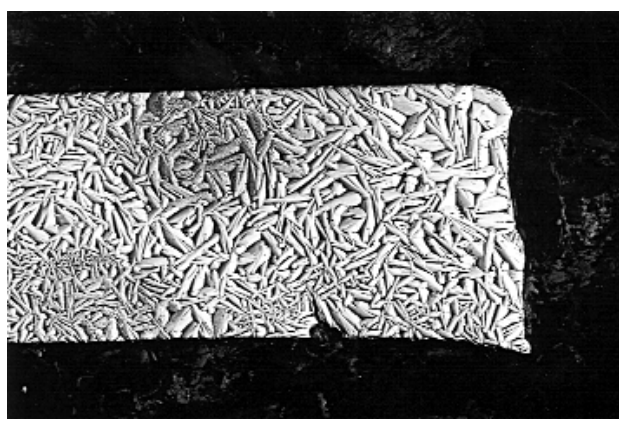


saltar também que dois conjuntos placa-parafuso apresentavam montagem com materiais dissimilares, prática não recomendada devido ao risco de corrosão pela formação de par galvânico. Finalmente, observou-se que o mecanismo de fratura da maioria dos implantes cirúrgicos e de todos os arames de Nitinol investigados estava relacionado ao fenômeno de corrosão do metal, que pode causar efeitos adversos no corpo humano.

A ABNT, através de seu Comitê Brasileiro Odonto-Médico-Hospitalar (CB26), vem realizando um grande esforço na elaboração/adaptação/tradução de normas técnicas para o setor. A globalização de padrões se tornou um assunto de importância crescente em países em desenvolvimento que estão estabelecendo suas indústrias de equipamentos médicos e agentes reguladores (Dorman-Smith, 2001; Yamac, 1998). Os documentos atualmente disponíveis na ABNT abrangem desde a especificação de matérias-primas e produtos, passando por requisitos de embalagem e marcação das peças, até aspectos dimensionais de componentes específicos (Tabela 4). Observou-se, no entanto, a ausência de versões nacionais de algumas normas importantes, como por exemplo, documentos que tratam das especificações da liga níquel-titânio de efeito memória para aplicações médicas (Nitinol) e dos procedimentos de retirada e análise de falha de implantes ci- rúrgicos (fabricados em metal, cerâmica e polímeros).

O trabalho de normatização é um requisito fundamental para a melhoria da qualidade dos produtos utilizados no Brasil. Apesar disso, ainda não há no país legislação sanitária moderna nem mecanismos reguladores que assegurem a qualidade dos implantes cirúrgicos em comercialização. Em 1996, os pesquisadores do INT já comentavam que a estruturação da certificação compulsória de produtos médico-odonto-hospitalares em aço inoxidável seria benéfica (Cavalcanti \& Coelho, 1997). Lastima-se que o serviço público de saúde, responsável por mais de $80 \%$ do consumo nacional de implantes cirúrgicos metálicos (ou aproximadamente R\$ 45 milhões) (SUS, 2001), não tenha criado até agora mecanismos técnicos para qualificar fornecedores durante seu processo de licitação, como forma de garantir a qualidade dos implantes cirúrgicos.

Além disso, não há no país legislação sanitária e mecanismos reguladores sobre os procedimentos de notificação e de análise a serem seguidos pelos hospitais, profissionais de saúde e fabricantes nos casos de falhas de implantes. Isso inviabiliza estudos estatísticos técnico-econômicos (principais causas, gastos públicos com reoperação, etc.) e, em alguns casos, prejudica as ações legais indenizatórias movidas por pacientes, seja pela documenta-

Tabela 4

Regras gerais da Associação Brasileira de Normas Técnicas de implantes cirúrgicos metálicos.

\begin{tabular}{lc}
\hline \multicolumn{1}{c}{ Normas para implantes em cirurgia } & Identificação \\
Título & NBR-ISO 8828 \\
\hline Orientações sobre cuidado e manuseio de implantes ortopédicos & NBR-ISO 6018 \\
Requisitos gerais para marcação, embalagem e rotulagem & NBR-ISO 14630 \\
Requisitos gerais & NBR-ISO 5832-1 \\
Aço inoxidável conformado & NBR-ISO 5832-2 \\
Titânio puro & NBR-ISO 5832-3 \\
Liga conformada de titânio 6-alumínio 4-vanádio & NBR-ISO 5832-4 \\
Liga fundida de cobalto-cromo-molibdênio & NBR-ISO 5832-5 \\
Liga conformada de cobalto-cromo-tungstênio-níquel & NBR-ISO 5832-6 \\
Liga conformada de cobalto-níquel-cromo-molibdênio & NBR-ISO 5832-7 \\
Liga forjada e conformada a frio de cobalto-cromo-níquel-molibdênio-ferro & NBR-ISO 5832-8 \\
Liga conformada de cobalto-níquel-cromo-molibdênio-tungstênio-ferro & NBR-ISO 5832-9 \\
Aço inoxidável conformado de alto nitrogênio & NBR-ISO 5832-10 \\
Liga conformada de titânio 5-alumínio 2,5-ferro & NBR-ISO 5832-11 \\
Liga conformada de titânio 6-alumínio 7-nióbio & NBR-ISO 5832-12 \\
Liga conformada de cobalto-cromo-molibdênio & NBR-ISO 13782 \\
Tântalo puro para aplicações em implantes cirúrgicos & No \\
\hline
\end{tabular}


ção insatisfatória do caso ou até mesmo, pelo extravio do implante metálico. Os casos de falha de implantes deveriam não somente ser notificados à agência reguladora (por exemplo, a Agência Nacional de Vigilância Sanitária, criada pelo Governo Federal em 1999), como também investigados minuciosamente por uma equipe multidisciplinar para verificar a conformidade do implante às suas especificações técnicas e identificar as causas da falha. Essa prática permitiria mapear a qualidade das diversas famílias de implantes cirúrgicos em comercialização no Brasil, além de criar um banco de dados com informações estatísticas sobre índices de falha, custos diretos e indiretos para o serviço público de saúde, etc. Essas informações forneceriam subsídios valiosos para a definição de medidas preventivas e de ações setoriais.

\section{Agradecimentos}

Os autores agradecem ao Eng. Tomaz Puga Leivas e ao Dr. Fernando Elias, ambos do Hospital das Clínicas, Faculdade de Medicina, Universidade de São Paulo, pela ajuda prestada. Este trabalho foi parcialmente financiado pela Fundação de Auxílio à Pesquisa do Estado de São Paulo (FAPESP) e pela Secretaria de Ciência, Tecnologia e Desenvolvimento Econômico do Estado de São Paulo.

\section{Conclusões}

- Nenhum dos materiais investigados obedece aos requisitos mínimos estabelecidos nas normas ABNT e ASTM (no caso de Nitinol, liga de níquel-titânio com efeito memória).

- Foram observados defeitos de fabricação, de projeto ou de montagem em alguns implantes investigados, que podem ter favorecido a sua falha prematura.

- O mecanismo de fratura da maioria dos componentes estava relacionado à corrosão do metal.

- Não há legislação sanitária nem mecanismos reguladores que tornem compulsórios os procedimentos de notificação dos casos de falhas de implantes cirúrgicos por profissionais de saúde, nem a investigação das causas da falha do componente, o que inviabiliza levantamentos estatísticos e ações setoriais.

\section{Referências}

ABIMO (Associação Brasileira da Indústria de Artigos e Equipamentos Médicos Odontológicos, Hospitalares e de Laboratórios), 2001. Histórico. Junho 2001 <http://www.abimo.org.br/historico.html>.

ABNT (Associação Brasileira de Normas Técnicas), 1999. Implantes para Cirurgia: Materiais Metálicos. Parte 1: Aço Inoxidável Comum. NBR-ISSO: 5832-1. Rio de Janeiro: ABNT.

ABNT (Associação Brasileira de Normas Técnicas), 1996. Implantes Ortopédicos: Requisitos Gerais para Marcação, Embalagem e Rotulagem. NBRISSO: 6018. Rio de Janeiro: ABNT.

ABNT (Associação Brasileira de Normas Técnicas), 1997a. Implantes para Cirurgia: Materiais Metálicos. Parte 2: Titânio Puro. NBR-ISSO: 5832-2. Rio Janeiro: ABNT. 
ABNT (Associação Brasileira de Normas Técnicas), 1997b. Implantes para Cirurgia: Materiais Metálicos, Parte 3: Liga Conformada de Titânio 6-Alumínio 4-Vanádio. NBR-ISSO: 5832-3. Rio de Janeiro: ABNT.

ANVISA (Agência Nacional de Vigilância Sanitária), 2001. Legislação, ANVISA-LEGIS. Junho $2001<\mathrm{http}$ : //www.anvisa.gov.br/legis/index.htm>.

ASTM (American Society for Testing and Materials), 1987. Standard Practice for Analysis of Retrieved Metallic Orthopedic Implants. ASTM: F561-87. Philadelphia: ASTM.

ASTM (American Society for Testing and Materials), 2000. Standard Specification for Wrought NickelTitanium Shape Memory Alloys for Medical Devices and Surgical Implants. ASTM: F2063-00. Philadelphia: ASTM.

BLACK, J., 1976. Implant retrieval: Problems and opportunities. In: Symposium on Retrieval and Analysis of Orthopedic Implants, Proceedings, NBS Special Publications 472, pp. 81-102. Gaithersburg: National Bureau Standards.

CAMPBELL, P.; MICKELLOP, H.; ALIM, R.; MIRRA, J.; NUTT, S.; DORR, L. \& AMSTUTZ, H. C., 1999. Metal-on-metal hip replacements: Wear performance and cellular response to wear particles. In: COBALT-BASE Alloys for Biomedical Applications (J. A. Disegi, R. L. Kennedy \& R. Pilliar, ed.), ASTM: STP 1365, pp. 193-209, Philadelphia: ASTM.

CASTLEMAN, L. S.; MOTZKIN, S. M.; ALICANDRI, F. P. \& BONAWIT, V. L., 1976. Biocompatibility of nitinol alloy as an implant material. Journal of Biomedical Materials Research, 10:695-731.

CAVALCANTI, E. C. \& COELHO, S., 1997. Implantes de aço inoxidável no caminho da regulamentação. Revista Inox, 5:6-8.

CAVALCANTI, E. C.; SOUZA, S. M. C. \& CAMPOS, M. M., 1995. Caracterização e avaliação da resistência à corrosão de implantes ortopédicos temporários removidos de pacientes. In: 18o Congresso Brasileiro de Corrosão, Anais, pp. 1001-1013. Rio de Janeiro: Associação Brasileira de Corrosão.

DORA, A.; LAW, F. C.; ALLEN, M. J. \& RUSHTON, N., 1998. Neoplastic transformation of cells by soluble but not particulate forms of metals in orthopaedic implants. Biomaterials, 9:751-759.

DORMAN-SMITH, V., 2001. Global harmonisation task force. In: Exporting Medical Devices to Europe: New Frontiers and Opportunities. Seminar Series, U.S. Department of Commerce. June 2001 <http://www.ita.doc.gov/td/mdequip/EUSeminarPresentations.html>

DUERIG, T.; PELTON, A. \& STÖCKEL, D., 1999. An overview of Nitinol medical applications. Materials Science and Engineering: A, 273-275:149160.

DUMBLENTON, J. H. \& MILLER, E. H., 1975. Failure of metallic orthopaedic implants. In: Metals Handbook (American Society for Metals - ASM, ed.), v. 10, pp. 571-580. 8th Ed. Metals Park: ASM International.

FDA (Food and Drugs Administration), 1996. Medical Device Reporting: An Overview. June $2001<\mathrm{http}$ // www.fda.gov/cdrh/mdr.html>.

FDA (Food and Drugs Administration), 2001. Recall of Zirconia Ceramic Femoral Heads for Hip Implants.
June $2001<$ http://www.fda.gov/cdrh/recalls zirconiahip.html>.

FERRAZ, O. L. M., 1997. Questionamentos judiciais e a proteção contra o paciente: Um sofisma a ser corrigido pelo gerenciamento de riscos. Revista Bioética do Conselho Federal de Medicina, v. 5, n. 1. Junho $2001<$ http://www.cfm.org.br/revista/ biolv5/questiona.html>.

ISO (International Organization for Standardization), 2000a. Retrieval and Analysis of Surgical Implants. Part 2: Analysis of Retrieved Metallic Surgical Implants. Geneva: ISO.

ISO (International Organization for Standardization), 1995. Implants for Surgery: Fundamental Principles. Geneva: ISO.

ISO (International Organization for Standardization), 1999. Retrieval and Analysis of Surgical Implants. Part 1: Retrieval and Handling. Geneva: ISO.

ISO (International Organization for Standardization), 2000b. Retrieval and Analysis of Surgical Implants. Part 3: Analysis of Retrieved Polymeric Surgical Implants. Geneva: ISO.

ISO (International Organization for Standardization), 2000c. Retrieval and Analysis of Surgical Implants. Part 4: Analysis of Retrieved Ceramic Surgical Implants. Geneva: ISO.

ITDS (International Trade Data System), 2001. Medical Equipment and Devices Industry. June 2001 $<$ http://www.itds.treas.gov/medicalequip.htm\# links $>$.

LEMON, T. T., 1977. Legal aspects of device retrieval. In: Symposium on Retrieval and Analysis of Orthopedic Implants, Proceedings, NBS Special Publication 472, pp. 73-80. Maryland: National Bureau of Standards.

MEARS, D. C., 1979. Materials and Orthopaedic Surgery. Baltimore: William \& Wilkins Company.

MDA (Medical Devices Agency), 1998. The Medical Devices Vigilance Systems. European Commission Guidelines. June $2001<\mathrm{http}$ // /www.medical-devices.gov.uk/mda-aic.htm\#report>.

MDA (Medical Devices Agency), 2001. DA 2001(05) Recall of Specified Batches of Zirconia Ceramic Femoral Heads for Use in Hip Replacements. MDA Adverse Incident Centre. June $2001<$ http:/ /www. medical-devices.gov.uk>.

NIH (National Institute of Health), 2000. Improving Medical Implant Performance Through Retrieval Information: Challenges and Opportunities. National Institute of Health Technology Assessment Conference Summary, U.S. Government. June 2001 <http://odp.od.nih.gov/consensus/ta/019/ 019_statement.htm>.

NISHIGAKI, M.; TANABE, A.; ITO, Y. \& MORIGUCHI, Y., 1980. Effect of hydride on the hydrogen embrittlement of CP titanium. In: International Conference on TI, Proceedings, pp. 1663-1670. Kyoto: American Institute of Mining, Metallurgical and Petroleum Engineers.

PIOTROWSKI, G., 1976. Clinical biomedics. In: Symposium on Retrieval and Analysis of Orthopedic Implants, Proceedings, NBS Special Publication 472, pp. 41-49, Maryland: National Bureau Standards.

POHLER, O. E. M., 1986. Failure of metallic orthopaedic implants. In: Metals Handbook (Ameri- 
can Society for Metals - ASM, ed.), v. 11, pp. 668694. $9^{\text {th }}$ Ed. Metals Parks: ASM International.

RAND-SCIENCE AND TECHNOLOGY POLICY INSTI-

TUTE. 2000. Biomaterials Availability: Potential Effects on Medical Innovation and Health Care. June 2001 <http://www.rand.org/publications/ ip/ip194/ip194.pdf>.

SCHUTZ, R. W. \& THOMAS, D. E., 1987. Corrosion of titanium and titanium alloys. In: Metals Handbook (American Society for Metals - ASM, ed.), v. 13, pp. 669-706. 9th Ed. Metals Parks: ASM International.

SCHWANINGER, B.; SARKAR, N. K. \& FOSTER, B. E., 1982. Effect of long term immersion corrosion on the flexural properties of nitinol. American Journal of Orthodontics, 81:45-49.

SMITH, G. K. \& BLACK, J., 1976. Models for systemic effects of metallic implants. In: Symposium on Retrieval and Analysis of Orthopedic Implants, Proceedings, NBS Special Publication 472, pp. 2330. Gaithersburg: National Bureau Standards.
SUS (Sistema Único de Saúde), 2001. Informações de Saúde: Procedimentos Hospitalares do SUS por Local de Internação. Conteúdo: Órtese e Prótese. Junho $2001<$ http:/ / www.datasus.gov.br>.

TORGENSEN, S. \& GJERDET, N. R., 1995. Retrieval Study of Stainless Steel and Titanium Miniplates and Screws Used in Maxillofacial Surgery. June 2001 <http://www.uib.no/cris/dok2>.

WILLIAMS, D. F., 1976. A review of metallurgical failure modes in orthopedic implants. In: Symposium on Retrieval and Analysis of Orthopedic Implants, Proceedings, NBS Special Publication 472, pp. 11-21. Gaithersburg: National Bureau of Standards.

YAMAC, T., 1998. Medical Devices: Materials for $\mathrm{Hu}$ man Contact. A Design Guide. London: Institute of Materials.

Recebido em 31 de agosto de 2001

Versão final reapresentada em 26 de janeiro de 2002 Aprovado em 25 de março de 2002 